\title{
Hyperpolarized Water to Study Protein-Ligand Interactions
}

\author{
Quentin Chappuis, ${ }^{\dagger}$ Jonas Milani, ${ }^{\dagger}$ Basile Vuichoud, ${ }^{\dagger}$ Aurélien Bornet, ${ }^{\dagger}$ Alvar D. Gossert, ${ }^{*}$, \\ Geoffrey Bodenhausen, ${ }^{\dagger, \S, \perp, \#}$ and Sami Jannin ${ }^{*},, \|$ \\ ${ }^{\dagger}$ Institut des Sciences et Ingénierie Chimiques, Ecole Polytechnique Fédérale de Lausanne (EPFL), 1015 Lausanne, Switzerland \\ ${ }^{\ddagger}$ Novartis Institutes for BioMedical Research, 4002 Basel, Switzerland \\ ${ }^{\S}$ Département de Chimie, Ecole Normale Supérieure, PSL, 75005 Paris, France \\ ${ }^{\perp}$ Sorbonne Université, UPMC Univ Paris 06, 75005 Paris, France \\ "Laboratoire des BioMolécules, UMR 7203, 75005 Paris, France \\ "Bruker BioSpin AG, 8117 Fällanden, Switzerland
}

Supporting Information

ABSTRACT: The affinity between a chosen target protein and small molecules is a key aspect of drug discovery. Screening by popular NMR methods such as Water-LOGSY suffers from low sensitivity and from false positives caused by aggregated or denatured proteins. This work demonstrates that the sensitivity of Water-LOGSY can be greatly boosted by injecting hyperpolarized water into solutions of proteins and ligands. Ligand binding can be detected in a few seconds, whereas about $30 \mathrm{~min}$ is usually required without hyperpolarization. Hyperpolarized water also enhances proton signals of proteins at concentrations below $20 \mu \mathrm{M}$ so that one can verify in a few seconds whether the proteins remain intact or have been denatured

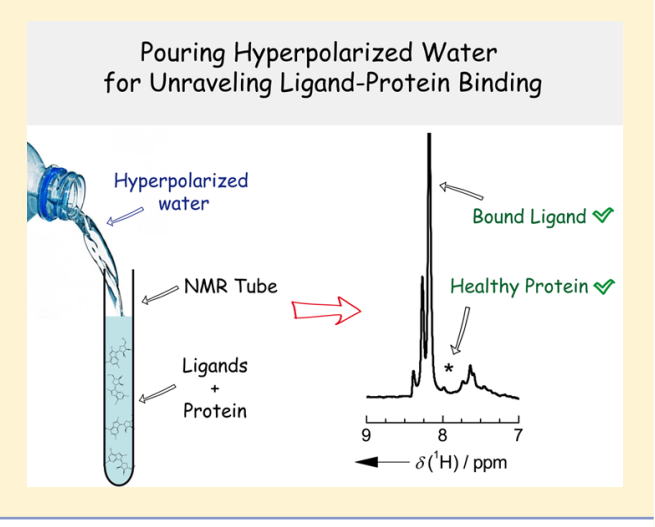

$\mathrm{D}$ rug discovery aspires to identify new lead compounds that bind with high affinity to target proteins or other biomolecules. Robust assays are required in order to determine binding affinities of extensive libraries of potential ligands. ${ }^{1,2}$ Two strategies for drug screening are mainly used nowadays, high-throughput screening (HTS) and fragment-based drug design (FBDD). In HTS, vast libraries of up to 2 million compounds are screened in order to find strong ligands (with dissociation constants $K_{\mathrm{D}}<10 \mu \mathrm{M}$ ) that can serve as lead compounds. On the other hand, FBDD seeks to identify small fragments, that is, compounds with molecular weights below $250 \mathrm{Da}$ that only weakly interact with the target proteins $(0.1<$ $\left.K_{\mathrm{D}}<10 \mathrm{mM}\right)$. Useful fragments found by primary screening in FBDD can then either be "expanded" chemically in so-called "fragment-growing" approaches or combined with other fragments in "fragment-linking" strategies to obtain new lead compounds.

Nuclear magnetic resonance (NMR) has become a wellestablished technique for both of these complementary strategies, albeit with distinct roles in HTS and FBDD approaches. For FBDD, NMR is used to screen libraries of carefully selected fragments and to characterize the weak interactions that are typical for such small compounds. ${ }^{3,4}$ In HTS campaigns on the other hand, NMR is employed to validate ligands identified by preliminary high-throughput assays. A HTS campaign can lead to a list of thousands of compounds. Such a hit-list contains "true" ligands that specifically bind to the target protein but also compounds that interfere with the high-throughput assay, for example, by causing the target protein to aggregate. These promiscuous compounds can usually only be identified by using expensive protein-observed NMR methods. Therefore, ideal NMR methods should be able to detect binding of very diverse ligands from HTS and FBDD approaches and, at the same time, monitor the integrity of the target protein in order to identify false positives.

A remarkable NMR experiment designed for the detection of interactions of proteins and ligands in solution has come to be known as Water-LOGSY (water-ligand observed via gradient spectroscopy). ${ }^{5-10}$ The detection of ligand binding by WaterLOGSY relies on the spontaneous transfer of polarization from water to ligands via either of two pathways, via the nuclear Overhauser effect (NOE) or via exchange of labile protons, ${ }^{6}$ though signals due to exchange are generally not considered as reliable reporters of binding. The transfer can either occur directly from the solvent to the ligand or be relayed indirectly via the bound protein. The sign of the NOE transfer depends on the rotational correlation time; rapidly tumbling free ligands acquire negative enhancements, while slowly tumbling ligandprotein complexes feature positive enhancements. Therefore,

Received: February 24, 2015

Accepted: April 15, 2015 
the difference between free and bound ligands can be readily identified. NMR signals observed in conventional WaterLOGSY experiments (without DNP) are typically 20-30 times weaker than signals in traditional one-dimensional NMR experiments because only magnetization transferred by NOE and/or exchange from water is selectively detected.

Hyperpolarization by dissolution dynamic nuclear polarization (D-DNP) $)^{11-14}$ is a method of choice to increase the nuclear spin polarization by $4-5$ orders of magnitude. D-DNP methods for drug screening have been developed recently by the group of Christian Hilty. These are based on the hyperpolarization of ${ }^{1} \mathrm{H}$ or ${ }^{19} \mathrm{~F}$ nuclei of ligands. ${ }^{15,16}$ Note that the enhanced polarization levels $P\left({ }^{1} \mathrm{H}\right)$ or $P\left({ }^{19} \mathrm{~F}\right)$ and corresponding relaxation times $T_{1}$ can vary significantly from ligand to ligand, leading to final signal enhancements that can differ by orders of magnitude. Here, we explore the combination of D-DNP and Water-LOGSY in view of highthroughput detection of protein-ligand interactions. Water is hyperpolarized by D-DNP in a comparable fashion to what has been shown by the groups of Jan-Henrik Ardenkjaer-Larsen ${ }^{17}$ and Lucio Frydman, ${ }^{18}$ but this could also be achieved by Overhauser liquid-state DNP. ${ }^{19-22}$ The hyperpolarization of water is highly reproducible and robust. The transfer of polarization from hyperpolarized water to the ligands is indicative of protein-ligand binding properties.

Hyperpolarization of ligands usually demands rapid proteinligand association after injection on a time scale that must be faster than the inevitable decay of the hyperpolarization by longitudinal $T_{1}$ relaxation. Hyperpolarization of water, on the other hand, allows one to probe protein-ligand equilibria even if the kinetics are very slow. Indeed, hyperpolarized water can be prepared and injected after the thermodynamic equilibrium of the protein-ligand interaction has been reached. As an additional benefit of water DNP, the spontaneous transfer of polarization from water to proteins enables the observation of their proton spectra ${ }^{18}$ even at concentrations below $20 \mu \mathrm{M}$. This offers a welcome means to check that the protein has remained intact. Results are reported for the protein Dot1L, a human histone methyl transferase, ${ }^{23,24}$ with both weakly and strongly binding ligands, typical of both FBDD and HTS approaches at various stages of drug discovery projects.

In the DNP-Water-LOGSY approach, a mixture of $\mathrm{H}_{2} \mathrm{O}$, a glassing agent such as glycerol, and a radical like TEMPOL is hyperpolarized at $\sim 1.2 \mathrm{~K}$ and $6.7 \mathrm{~T}^{13}$ with microwave frequency modulation. ${ }^{25}$ The hyperpolarized sample is then quickly dissolved using superheated $\mathrm{D}_{2} \mathrm{O}$ and transferred to an NMR magnet through a magnetic tunnel, ${ }^{26}$ where it is rapidly injected into an equilibrated protein-ligand solution (Figure 1). The excitation of hyperpolarized water can lead to massive radiation damping (RD) and demagnetizing distant dipolar field effects. ${ }^{27}$ Therefore, frequency-selective pulses are used in order to preserve the water resonance while detecting ligand and protein signals with full sensitivity (see the Supporting Information for details on the preparation of hyperpolarized water and on the adaptation of the Water-LOGSY experiment).

Direct Detection of Weak Binders. The protein Dot $1 \mathrm{~L}$ and its weak ligand adenosine were chosen as a model system to demonstrate the potential of DNP-Water-LOGSY (see the Supporting Information for details on the preparation of Dot1L). Dot1L is a histone methyl transferase enzyme, that is, it methylates histone proteins around which DNA is tightly wrapped in the cell nucleus. ${ }^{28}$ Such methylation events of histones can modulate the packing and accessibility of DNA for (a) Illustration of the experimental method

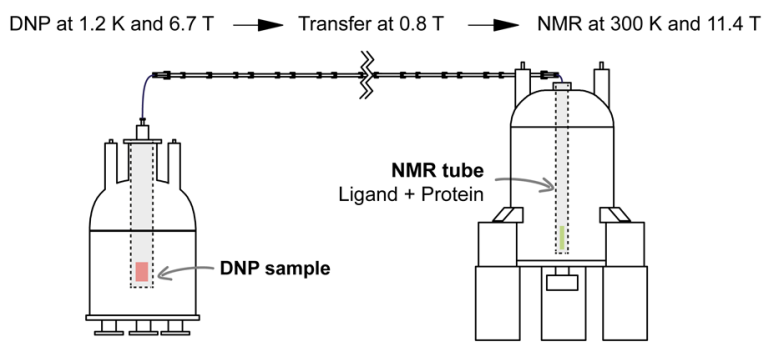

(b) Water hyperpolarization followed by DNP-Water-LOGSY
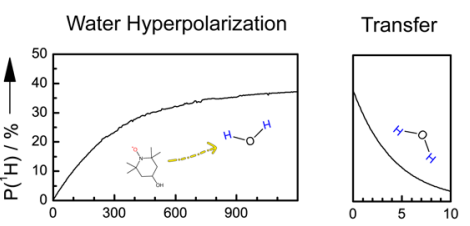

DNP-WaterLOGSY

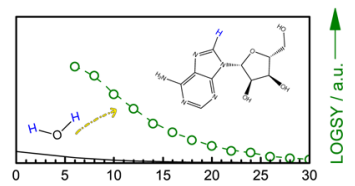

time $/ \mathrm{s} \longrightarrow$

Figure 1. (a) Schematic apparatus for DNP-Water-LOGSY experiments. (b) (left) Solid-state NMR signal of the DNP buildup of the proton polarization $P\left({ }^{1} \mathrm{H}\right)$ of water. A typical polarization $P\left({ }^{1} \mathrm{H}\right)>$ $38 \%$ can be achieved with TEMPOL as the polarizing agent and glycerol as the glass-forming agent. (middle) Sample dissolution, transfer through a "magnetic tunnel", and injection into an NMR tube containing a mixture of a target protein such as Dot1L plus one or more ligands like adenosine. (right) Liquid-state NMR signals of the hyperpolarized water and adenosine. The water polarization is transferred through NOE and/or chemical exchange in the manner of Water-LOGSY.

transcription and therefore influence cellular function. Dot1L plays a role in embryonic development and hematopoiesis, but aberrant activity can lead to leukemia. ${ }^{23}$ Mechanistically, Dot1L transfers a methyl group of the tightly bound $\left(K_{\mathrm{D}}=300-500\right.$ $\mathrm{nM})$ cofactor S-(5'-adenosyl)-L-methionine (SAM) onto lysine 79 of histone $\mathrm{H} 3$. The protein-ligand system used in the present work consists of Dot1L (residues $2-332,38 \mathrm{kDa}$ ) and adenosine, which is a fragment of SAM that binds weakly to $\operatorname{Dot} 1 \mathrm{~L}\left(K_{\mathrm{D}}=100 \mu \mathrm{M}\right.$; concentrations after dilution [Dot1L] = $21.5 \mu \mathrm{M}$ and [adenosine] $=2.15 \mathrm{mM}$, that is, a 100 -fold excess). The intense longitudinal water magnetization generated by D-DNP (Figure 2a) is partially transferred to adenosine, either directly or indirectly via the protein, so that the ligand is also hyperpolarized (Figure $2 \mathrm{~b}$ ). In the absence of protein (Figure 3a $(1)$ ), all adenosine molecules are in their free form with a rotational correlation time $\tau_{\mathrm{c}} \ll 1 / \omega_{0}$ so that the signals of both nonexchangeable aromatic protons $\mathrm{H}^{2}$ and $\mathrm{H}^{8}$ are negatively enhanced. In the presence of protein (Figure 3a (2)), adenosine binds in the fast exchange regime so that, even though adenosine is in a 100 -fold excess with respect to the protein, the signals of the nonexchangeable adenosine protons are positively enhanced because the complex has a rotational correlation time $\tau_{\mathrm{c}}>1 / \omega_{0}$. DNP-Water-LOGSY thus allows one to reveal the binding of a weak ligand unambiguously.

Detection of Strong Binders by Competition. Strong binders can be detected indirectly by competition experiments when a weak binder is used as a "spy ligand". 29,30 The strong binders displace the spy ligand to its free form, hence giving rise to negative LOGSY signals of the weak binder that indicate its displacement by the stronger ligand. The two natural ligands SAM and S-(5'-adenosyl)-L-homocysteine (SAH) (see Figure $3 \mathrm{~b})$ bind to 
(a) Hyperpolarized water decay

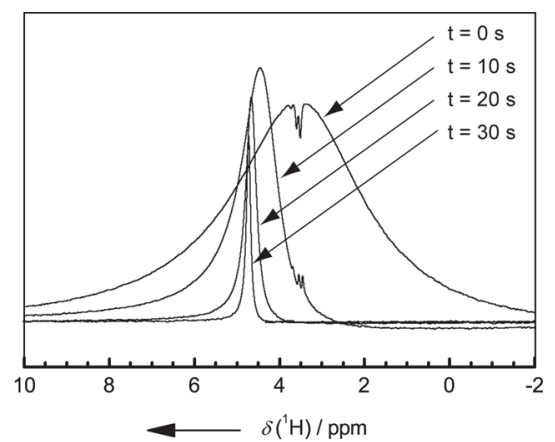

(b) DNP-Water-LOGSY of adenosine bound to Dot1L

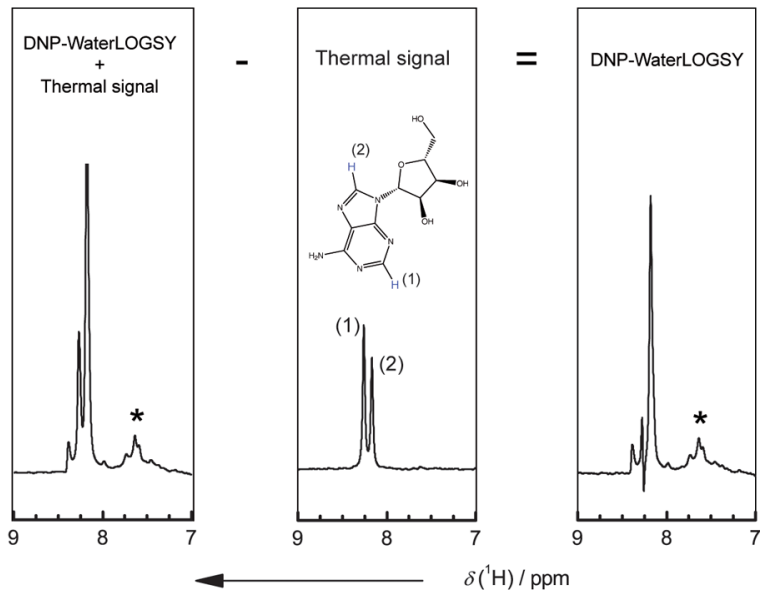

Figure 2. (a) Water signals after dissolution, monitored by $0.01^{\circ}$ excitation pulses. Initially, the water signal is strongly broadened by Radiation Damping $\mathrm{RD}$. As the polarization decays, the integral decreases while the lines become narrower. (b) DNP-Water-LOGSY signals of the aromatic protons of adenosine measured in the presence of $21.5 \mu \mathrm{M}$ of the protein Dot $1 \mathrm{~L}$ at $500 \mathrm{MHz}$ and $298 \mathrm{~K}$. The DNPenhanced spectrum (left) arises from the magnetization transferred from water to the ligand but also from the magnetization of the ligand in thermal equilibrium. A spectrum measured after complete relaxation (center) can be subtracted from the DNP-enhanced spectrum so that the difference only contains pure DNP-enhanced Water-LOGSY information (right). The hyperpolarized signals of the protein are highlighted by $(*)$.

Dot1L with strong affinities $\left(K_{\mathrm{D}}=300-500\right.$ and $100 \mathrm{nM}$, respectively) associated with slow kinetics. When added to the adenosine/Dot1L mixture prior to injection of hyperpolarized water, these strong binders displace adenosine from the protein and therefore invert the sign of the LOGSY signals of adenosine after injection. It was indeed possible to observe a partial displacement of adenosine by stronger competitors such as SAM or SAH. Figure 3a (3) shows how the addition of 0.1 mM SAM (i.e., with adenosine in 20 -fold excess with respect to SAM) causes a partial displacement of adenosine, while the addition of $0.7 \mathrm{mM} \mathrm{SAM}$ (i.e., with adenosine in 3-fold excess) causes its complete displacement (Figure 3a (4)). Knowing the affinity of the spy ligand and the relative concentrations of the spy and strong ligands, dissociation constants can be determined by titration. Adenosine is more efficiently displaced by the addition of $0.1 \mathrm{mM}$ SAH than by SAM in equal amounts, thus confirming that SAH has a higher affinity for Dot1L than SAM (Figure 3a (5)). In this manner, relative binding strengths of strong ligands can be determined even if (a) DNP-Water-LOGSY competition experiments

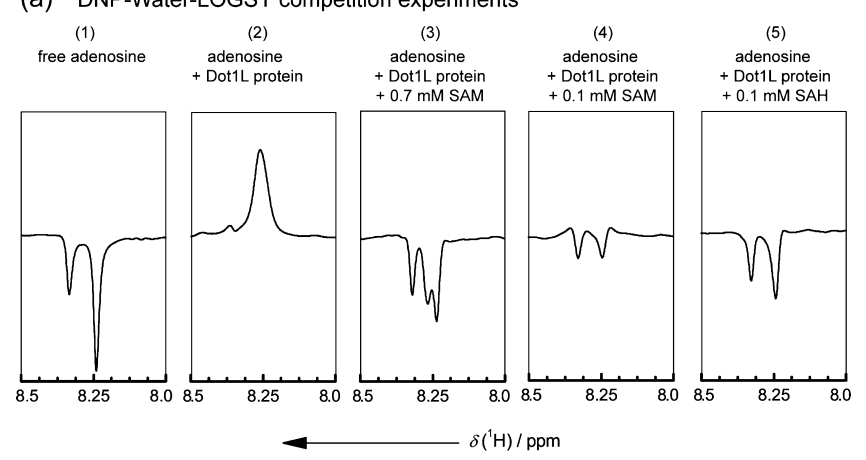

(b) Molecular structure of SAM and SAH
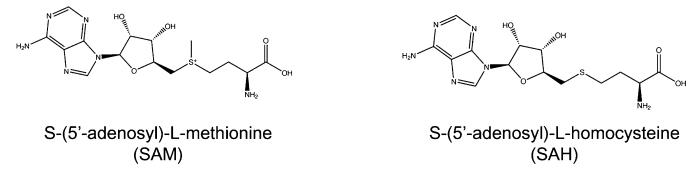

Figure 3. (a) Water-LOGSY signals of the two aromatic protons of adenosine, which binds weakly to the protein Dot1L. The thermal equilibrium signals were subtracted as described in Figure 2. (1) In the absence of protein, free adenosine yields negative LOGSY signals. (2) In the presence of protein, bound adenosine yields positive LOGSY signals. Stronger competing binders displace adenosine from the binding site of Dot1L, so that negative LOGSY signals are seen, characteristic of the free ligand, in the presence of (3) $0.7 \mathrm{mM}$ SAM and (4) $0.1 \mathrm{mM}$ SAM or (5) $0.1 \mathrm{mM} \mathrm{S}$-(5'-adenosyl)-L-homocysteine (SAH). (b) Molecular structures of SAM and SAH.

they are in slow exchange, provided that the equilibrium is established between SAM and adenosine-bound forms of Dot1L prior to the injection of hyperpolarized water. Due to the slow kinetics of SAM binding to Dot1L, it may take several seconds or minutes to establish this equilibrium. If one chooses to hyperpolarize the ligands rather than water, such slow equilibria cannot be studied. With DNP-Water-LOGSY, even very slow equilibria can be probed, enabling the determination of $K_{\mathrm{D}}$ despite slow kinetics. DNP-Water-LOGSY can therefore be applied to more advanced stages of drug discovery where such situations frequently occur. In principle, the direct detection of strong binders is also possible with DNP-WaterLOGSY. This will be the subject of further studies.

Detecting Hyperpolarized Proton Signals of Proteins. Protein aggregation is a major source of false positive results in WaterLOGSY experiments. Protein aggregates often expose hydrophobic surfaces where ligands can bind in a nonspecific manner. In conventional Water-LOGSY experiments, this leads to a misleading indirect (positive) magnetization transfer and therefore to false positive results. A useful secondary benefit of our DNP-Water-LOGSY approach is the partial hyperpolarization of the protein. ${ }^{18}$ This allows one to verify the integrity of the protein in real time and to check that it has not been denatured. Figure 4a shows the time course of protein signals in a DNP-Water-LOGSY experiment. The efficiency of the transfer of hyperpolarized magnetization from water to the protein was even greater than the transfer to the ligands. The protein signals were enhanced by a factor of about 20 with respect to their thermal equilibrium. In order to illustrate the usefulness of this feature in drug discovery experiments, a DNP-Water-LOGSY experiment was performed using Dot1L that was denatured by heating to $100{ }^{\circ} \mathrm{C}$ for $5 \mathrm{~min}$. With the resulting aggregated protein, a misleading positive LOGSY 
(a) Protein hyperpolarization decay

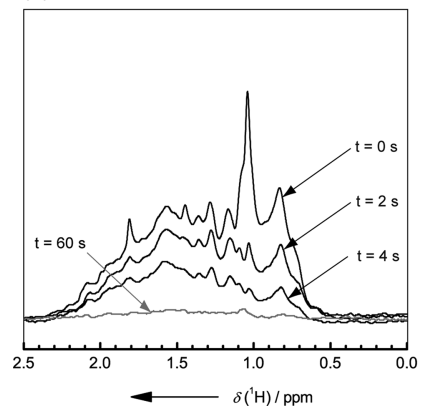

(b) Protein control: intact or denatured

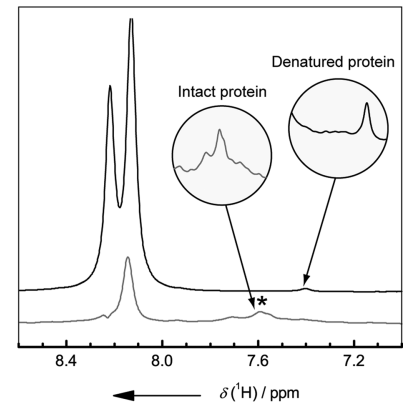

Figure 4. Transfer of proton hyperpolarization from water to the protein Dot1L. (a) Hyperpolarized proton signals in the methyl region of the protein Dot1L $(21.5 \mu \mathrm{M}$ at $298 \mathrm{~K}$ and $500 \mathrm{MHz})$, observed by DNP-Water-LOGSY. The polarization is transferred from hyperpolarized water of the protons of the protein by NOE and exchange of labile protons. The protein signals decay with the longitudinal relaxation time $T_{1}\left({ }^{1} \mathrm{H}\right)=4.2 \mathrm{~s}$ of water. (b) LOGSY spectra of 2.15 $\mathrm{mM}$ adenosine in the presence of $21.5 \mu \mathrm{M}$ folded Dot $1 \mathrm{~L}$ (gray curve) and after denaturation by heating to $100{ }^{\circ} \mathrm{C}$ for $5 \mathrm{~min}$ (black curve.) The intense positive adenosine signal is evidence for binding to Dot $1 \mathrm{~L}$. The expanded amide region between 7.8 and $7.2 \mathrm{ppm}$ reveals the state of integrity or degradation of the protein. DNP-Water-LOGSY thus allows one to discard false positive results due to protein denaturation.

signal was obtained for adenosine (five times stronger than with the intact folded protein). However, the protein signals recorded with the same experiment were distinctly different, thereby revealing extensive degradation of the protein (Figure $4 \mathrm{~b})$. Thus, false positive results of DNP-Water-LOGSY due to protein degradation can be readily identified, conferring a very high robustness to this methodology. The high signal-to-noise ratio obtained at a protein concentration of only $21.5 \mu \mathrm{M}$ Dot $1 \mathrm{~L}$ suggests that concentrations as low as $1 \mu \mathrm{M}$ should be accessible.

We are currently taking steps to build an injection system ${ }^{31}$ to reduce the transfer and injection time below $2 \mathrm{~s}$, which will boost the DNP enhancement of water after transfer. The separation of radicals ${ }^{18}$ would also provide a net improvement by suppressing paramagnetic relaxation. Another promising route is the use of hybrid polarizing materials (HYPSO) ${ }^{32}$ that would have to be optimized for the hyperpolarization of water. The Water-LOGSY technique is particularly well suited for DNP, especially in terms of reproducibility. Regardless of the variety of ligands and proteins under investigation, only water needs to be hyperpolarized. This greatly simplifies the D-DNP experiments. By contrast, if different ligands are to be hyperpolarized, one has to take into account variations in their enhancements and relaxation rates. Furthermore, one needs not fear that any ligands could precipitate in the magnetic tunnel during transfer, so that there is no risk of clogging the transfer line. Another major advantage of Water-LOGSY is the high contrast between binding and nonbinding ligands because their signals have opposite signs, while other techniques require comparing line widths or lifetimes or rely on subtle differences between pairs of spectra. ${ }^{33}$ The distinction of negative and positive signals is not hampered by $B_{0}$-field inhomogeneity that can be associated with D-DNP.

In summary, DNP-Water-LOGSY enables simple and robust hyperpolarized drug screening experiments because only water needs to be hyperpolarized. The method can be applied to a broad range of situations relevant for drug discovery, and it is highly robust with respect to artifacts. The direct detection of weak binders by DNP-Water-LOGSY is particularly well suited for primary screening in FBDD. On the other hand, if performed in competition mode, DNP-Water-LOGSY can also be applied to confirm "hits" in HTS campaigns, where one seeks to identify strong interactions. In addition to drug screening, the transfer of hyperpolarized magnetization from water to proteins can be used for various studies of proteins. This feature provides DNP-Water-LOGSY with a unique advantage over other ligand-based screening techniques. Because of the efficient polarization transfer from the solvent to the protein, the integrity of the protein can be determined, and false positives due to nonspecific binding to aggregated proteins can be discarded.

\section{ASSOCIATED CONTENT}

\section{S Supporting Information}

Additional details are supplied as Supporting Information: (1) the preparation of hyperpolarized water, (2) the adaptation of Water-LOGSY experiments to D-DNP, and (3) preparation of the protein Dot1L. This material is available free of charge via the Internet at http://pubs.acs.org.

\section{AUTHOR INFORMATION}

\section{Corresponding Authors}

*E-mail: sami.jannin@epfl.ch (S.J.).

*E-mail: alvar.gossert@novartis.ch (A.D.G.).

\section{Notes}

The authors declare no competing financial interest.

\section{ACKNOWLEDGMENTS}

We would like to thank Marcel J. J. Blommers from Novartis for helpful discussions, and Anto Barisic and Dr. Pascal Miéville from EPFL for valuable assistance. This work was supported by the Swiss National Science Foundation (SNSF), the Ecole Polytechnique Fédérale de Lausanne (EPFL), the Swiss Commission for Technology and Innovation (CTI), Bruker BioSpin Switzerland, the CNRS, and the European Research Council (ERC), Grant Agreement 339754 “Dilute para-water”.

\section{REFERENCES}

(1) Dalvit, C. NMR Methods in Fragment Screening: Theory and a Comparison with Other Biophysical Techniques. Drug Discovery Today 2009, 14, 1051-1057.

(2) Schulz, M. N.; Hubbard, R. E. Recent Progress in FragmentBased Lead Discovery. Curr. Opin. Pharmacol. 2009, 9, 615-621.

(3) Salvi, N.; Buratto, R.; Bornet, A.; Ulzega, S.; Rentero Rebollo, I.; Angelini, A.; Heinis, C.; Bodenhausen, G. Boosting the Sensitivity of Ligand-Protein Screening by NMR of Long-Lived States. J. Am. Chem. Soc. 2012, 134, 11076-11079.

(4) Buratto, R.; Bornet, A.; Milani, J.; Mammoli, D.; Vuichoud, B.; Salvi, N.; Singh, M.; Laguerre, A.; Passemard, S.; Gerber-Lemaire, S.; Jannin, S.; Bodenhausen, G. Drug Screening Boosted by Hyperpolarized Long-Lived States in NMR. Chem. Med. Chem. 2014, 9, 2509-2515.

(5) Dalvit, C. Homonuclear 1D and 2D NMR Experiments for the Observation of Solvent-Solute Interactions. J. Magn. Reson., Ser. B 1996, 112, 282-288.

(6) Dalvit, C.; Fogliatto, G.; Stewart, A.; Veronesi, M.; Stockman, B. WaterLOGSY as a Method for Primary NMR Screening: Practical Aspects and Range of Applicability. J. Biomol. NMR 2001, 21, 349359.

(7) Dalvit, C.; Pevarello, P.; Tato, M.; Veronesi, M.; Vulpetti, A.; Sundstrom, M. Identification of Compounds with Binding Affinity to 
Proteins via Magnetization Transfer from Bulk Water. J. Biomol. NMR 2000, 18, 65-68.

(8) Furihata, K.; Shimotakahara, S.; Tashiro, M. An Efficient Use of the WATERGATE W5 Sequence for Observing a Ligand Binding with a Protein Receptor. Magn. Reson. Chem. 2008, 46, 799-802.

(9) Gossert, A. D.; Henry, C.; Blommers, M. J. J.; Jahnke, W.; Fernandez, C. Time Efficient Detection of Protein-Ligand Interactions with the Polarization Optimized PO-WaterLOGSY NMR Experiment. J. Biomol. NMR 2009, 43, 211-217.

(10) Liu, M. L.; Mao, X. A.; Ye, C. H.; Huang, H.; Nicholson, J. K.; Lindon, J. C. Improved WATERGATE Pulse Sequences for Solvent Suppression in NMR Spectroscopy. J. Magn. Reson. 1998, 132, 125129.

(11) Abragam, A.; Goldman, M. Principles of Dynamic NuclearPolarization. Rep. Prog. Phys. 1978, 41, 395-467.

(12) Ardenkjaer-Larsen, J. H.; Fridlund, B.; Gram, A.; Hansson, G.; Hansson, L.; Lerche, M. H.; Servin, R.; Thaning, M.; Golman, K. Increase in Signal-to-Noise Ratio of $>10,000$ Times in Liquid-State NMR. Proc. Natl. Acad. Sci. U.S.A. 2003, 100, 10158-10163.

(13) Jannin, S.; Bornet, A.; Melzi, R.; Bodenhausen, G. High Field Dynamic Nuclear Polarization at $6.7 \mathrm{~T}$ : Carbon-13 Polarization above 70\% within $20 \mathrm{~min}$. Chem. Phys. Lett. 2012, 549, 99-102.

(14) Mieville, P.; Ahuja, P.; Sarkar, R.; Jannin, S.; Vasos, P. R.; Gerber-Lemaire, S.; Mishkovsky, M.; Comment, A.; Gruetter, R.; Ouari, O.; Tordo, P.; Bodenhausen, G. Scavenging Free Radicals to Preserve Enhancement and Extend Relaxation Times in NMR Using Dynamic Nuclear Polarization. Angew. Chem., Int. Ed. 2010, 49, 78347834.

(15) Lee, Y.; Zeng, H. F.; Mazur, A.; Wegstroth, M.; Carlomagno, T.; Reese, M.; Lee, D.; Becker, S.; Griesinger, C.; Hilty, C. Hyperpolarized Binding Pocket Nuclear Overhauser Effect for Determination of Competitive Ligand Binding. Angew. Chem., Int. Ed. 2012, 51, 51795182.

(16) Lee, Y.; Zeng, H. F.; Ruedisser, S.; Gosser, A. D.; Hilty, C. Nuclear Magnetic Resonance of Hyperpolarized Fluorine for Characterization of Protein-Ligand Interactions. J. Am. Chem. Soc. 2012, 134, 17448-17451.

(17) Ardenkjaer-Larsen, J. H.; Laustsen, C.; Bowen, S.; Rizi, R. Hyperpolarized $\mathrm{H}_{2} \mathrm{O}$ MR Angiography. Magn. Reson. Med. 2014, 71, $50-56$.

(18) Harris, T.; Szekely, O.; Frydman, L. On the Potential of Hyperpolarized Water in Biomolecular NMR Studies. J. Phys. Chem. B 2014, 118, 3281-3290.

(19) Overhauser, A. W. Polarization of Nuclei in Metals. Phys. Rev. 1953, 92, 411-415.

(20) McCarney, E. R.; Armstrong, B. D.; Lingwood, M. D.; Han, S. Hyperpolarized Water As an Authentic Magnetic Resonance Imaging Contrast Agent. Proc. Natl. Acad. Sci. U.S.A. 2007, 104, 1754-1759.

(21) Neugebauer, P.; Krummenacker, J. G.; Denysenkov, V. P.; Parigi, G.; Luchinat, C.; Prisner, T. F. Liquid State DNP of Water at 9.2 T: An Experimental Access to Saturation. Phys. Chem. Chem. Phys. 2013, 15, 6049-6056.

(22) Armstrong, B. D.; Han, S. G. Overhauser Dynamic Nuclear Polarization to Study Local Water Dynamics. J. Am. Chem. Soc. 2009, 131, 4641-4647.

(23) Okada, Y.; Feng, Q.; Lin, Y. H.; Jiang, Q.; Li, Y. Q.; Coffield, V. M.; Su, L. S.; Xu, G. L.; Zhang, Y. hDOT1L Links Histone Methylation to Leukemogenesis. Cell 2005, 121, 167-178.

(24) Jones, B.; Su, H.; Bhat, A.; Lei, H.; Bajko, J.; Hevi, S.; Baltus, G. A.; Kadam, S.; Zhai, H. L.; Valdez, R.; Gonzalo, S.; Zhang, Y.; Li, E.; Chen, T. P. The Histone H3K79 Methyltransferase Dot1L Is Essential for Mammalian Development and Heterochromatin Structure. Plos Genet. 2008, 4, e1000190.

(25) Bornet, A.; Milani, J.; Vuichoud, B.; Perez Linde, A. J.; Bodenhausen, G.; Jannin, S. Microwave Frequency Modulation to Enhance Dissolution Dynamic Nuclear Polarization. Chem. Phys. Lett. 2014, 602, 63-67.
(26) Milani, J.; Vuichoud, B.; Bornet, A.; Mieville, P.; Mottier, R.; Jannin, S.; Bodenhausen, G. A Magnetic Tunnel to Shelter Hyperpolarized Fluids. Rev. Sci. Instrum. 2015, 86, 024101.

(27) Jeener, J. Dipolar Field and Radiation Damping: Collective Effects in Liquid-State NMR. In eMagRes; John Wiley \& Sons, Ltd: New York, 2007.

(28) Lacoste, N.; Utley, R. T.; Hunter, J. M.; Poirier, G. G.; Cote, J. Disruptor of Telomeric Silencing-1 Is a Chromatin-Specific Histone H3 Methyltransferase. J. Biol. Chem. 2002, 277, 30421-30424.

(29) Dalvit, C.; Flocco, M.; Knapp, S.; Mostardini, M.; Perego, R.; Stockman, B. J.; Veronesi, M.; Varasi, M. High-Throughput NMRBased Screening with Competition Binding Experiments. J. Am. Chem. Soc. 2002, 124, 7702-7709.

(30) Jahnke, W.; Floersheim, P.; Ostermeier, C.; Zhang, X.; Hemmig, R.; Hurth, K.; Uzunov, D. P. NMR Reporter Screening for the Detection of High-Affinity Ligands. Angew. Chem., Int. Ed. 2002, 41, $3420-3423$.

(31) Bowen, S.; Hilty, C. Rapid Sample Injection for Hyperpolarized NMR Spectroscopy. Phys. Chem. Chem. Phys. 2010, 12, 5766-5770.

(32) Gajan, D.; Bornet, A.; Vuichoud, B.; Milani, J.; Melzi, R.; van Kalkeren, H. A.; Veyre, L.; Thieuleux, C.; Conley, M. P.; Gruning, W. R.; Schwarzwalder, M.; Lesage, A.; Coperet, C.; Bodenhausen, G.; Emsley, L.; Jannin, S. Hybrid Polarizing Solids for Pure Hyperpolarized Liquids through Dissolution Dynamic Nuclear Polarization. Proc. Natl. Acad. Sci. U.S.A. 2014, 111, 14693-14697.

(33) Hajduk, P. J.; Olejniczak, E. T.; Fesik, S. W. One-Dimensional Relaxation- and Diffusion-Edited NMR Methods for Screening Compounds that Bind to Macromolecules. J. Am. Chem. Soc. 1997, 119, 12257-12261. 